\title{
Multivariate meta-analysis of multiple outcomes: characteristics and predictors of borrowing of strength from Cochrane reviews
}

Miriam Hattle ( $\square$ m.s.hattle@keele.ac.uk)

Keele University https://orcid.org/0000-0003-1542-6277

Danielle L. Burke

Keele University School of Medicine

Thomas Trikalinos

Brown University School of Public Health

Christopher H. Schmid

Brown University School of Public Health

\section{Yong Chen}

University of Pennsylvania Perelman School of Medicine

\section{Dan Jackson}

AstraZeneca UK Ltd

Richard D. Riley

Keele University School of Medicine

\section{Research}

Keywords: Meta-analysis, Borrowing of strength, Multivariate meta-analysis, IPD meta-analysis

Posted Date: September 21st, 2021

DOI: https://doi.org/10.21203/rs.3.rs-916711/v1

License: (c) (7) This work is licensed under a Creative Commons Attribution 4.0 International License. Read Full License 


\section{Abstract \\ Objectives}

Multivariate meta-analysis allows the joint synthesis of multiple outcomes accounting for their correlation. This enables borrowing of strength (BoS) across outcomes, which may lead to greater efficiency and even different conclusions compared to separate univariate meta-analyses. However, multivariate meta-analysis is complex to apply, so guidance is needed to flag (in advance of analysis) when the approach is most useful.

\section{Study design and setting}

We use 43 Cochrane intervention reviews to empirically investigate the characteristics of meta-analysis datasets that are associated with a larger BoS statistic (from 0 to 100\%) when applying a bivariate meta-analysis of binary outcomes.

\section{Results}

Four characteristics were identified as strongly associated with BoS. the total number of studies, the number of studies with the outcome of interest, the percentage of studies missing the outcome of interest, and the largest absolute within-study correlation. Using these characteristics, we then develop a model for predicting BoS in a new dataset, which is shown to have good performance (an adjusted $\mathrm{R}^{2}$ of $50 \%$ ). Applied examples are used to illustrate the use of the BoS prediction model.

\section{Conclusions}

Cochrane reviewers currently use univariate meta-analysis methods, but our prediction model for BoShelps to flag when a multivariate meta-analysis may also be beneficial in Cochrane reviews with multiple binary outcomes. Extension to non-Cochrane reviews and other outcome types is still required.

\section{Introduction}

Conventional methods for meta-analysis produce a single summary result, for example about one particular outcome. In particular, in Cochrane intervention reviews an inverse-variance weighting meta-analysis is typically applied to each outcome of interest separately, and so each meta-analysis utilises just one intervention effect estimate per study. This process can be coined a univariate meta-analysis, with the word 'univariate' indicating a single summary result is of interest. However, most meta-analysis projects aim to produce multiple summary results, especially because multiple outcomes are of interest, such as a hypertensive participant's systolic (SBP) and diastolic (DBP) blood pressure, a migraine sufferer's levels of pain and nausea, a cancer participant's disease-free and overall survival times, and pregnancy outcomes for both the mother and her baby [1]. This potentially motivates a multivariate meta-analysis, to produce multiple summary results (one for each outcome) jointly from the same meta-analysis model [2].

The key advantage of a multivariate meta-analysis of multiple outcomes is to account for their correlation [3]. At the participant-level, multiple health outcomes are often correlated with each other, and this leads to correlation amongst 
multiple effect estimates from the same study. Such correlation of a pair of effect estimates is known as withinstudy correlation. For example, in a randomised trial of anti-hypertensive treatment, the estimated treatment effects for SBP and DBP are likely to have a positive within-study correlation, caused by a positive correlation at the participant-level between SBP and DBP. When there is between-study heterogeneity in effects there may also be between-study correlation, which arises when the true effect for each outcome is correlated with the true effect for another outcome. For example, the true effect of anti-hypertensive treatment on SBP usually has a positive betweenstudy correlation with the true effect on DBP, caused by changes in study and participant characteristics (such as dose and mean blood pressure at baseline) which modify the true treatment effects on SBP and DBP in the same direction.

Accounting for outcome correlation in a multivariate meta-analysis allows more studies to contribute toward the meta-analysis results for each outcome (i.e. a study can be included in the analysis even though it has not measured all the outcomes of interest), which may improve efficiency and even decrease bias (e.g. due to selective outcome reporting [4]) compared to undertaking a separate univariate meta-analysis for each outcome. In particular, alongside any direct evidence, the multivariate meta-analysis allows the summary result for each outcome to depend on correlated results from other outcomes. The rationale is that by observing information from related outcomes we can learn something about the missing outcomes of interest, and thus gain some knowledge that is otherwise lost; a concept known statistically as borrowing of strength (BoS) $[5,6]$. However, a downside of multivariate meta-analysis is that the approach is more complex than undertaking separate univariate meta-analyses (one for each outcome), as it requires the meta-analyst to obtain or derive within-study correlations between pairs of treatment effect estimates in the same study $[7,8]$. Furthermore, the amount of borrowing of strength is often small. Trikalinos et al. examined 45 Cochrane reviews that contained two or three binary outcomes that could be analysed using either univariate or multivariate meta-analysis $[9,10]$. They conclude that if the "focus is on the summary effects and the confidence intervals then the choice between the univariate and multivariate meta-analysis has limited practical importance"[9]. Yet, isolated examples within the Trikalinos review do exhibit important differences between univariate and multivariate meta-analysis, and other examples exist where multivariate meta-analysis has an important impact [3, 11-14].

Guidance is therefore needed to help researchers identify (in advance of actual analysis) whether a multivariate meta-analysis may be useful in their particular review. To address this, in this article we use the set of Cochrane reviews identified by Trikalinos et al. to investigate the characteristics of meta-analysis datasets that are associated with larger BoS when applying a multivariate meta-analysis. We then derive a multivariable prediction model for predicting the amount of BoS conditional on these characteristics, which can be applied to flag when to use the multivariate approach in practice. Note that we focus on the benefits of multivariate meta-analysis for estimating the summary effect for each outcome, and do not consider functions of the outcomes (e.g. differences in summary effect for outcomes 1 and 2), as then the benefit of a multivariate meta-analysis to account for correlation is more obvious (i.e. to determine the correct variance of the function) [15].

\section{Methods}

We firstly introduce the Trikalinos dataset to be used through the article. Then we describe our methods to identify characteristics associated with BoS, and for developing a multivariable prediction model for BoS. 


\section{Data analysis using 43 reviews of binary outcomes identified by Trikalinos}

There were 45 reviews included in the empirical review by Trikalinos et al $[9,10]$. Each review contained at least seven studies that reported both outcomes or at least half the studies with both outcomes if the total number of studies was greater than 14. Each of the studies satisfying the previous requirement (i.e. at least seven studies with both outcomes) had at least 10 patients and at least two events. There were two reviews that contained three outcomes, but these were excluded for our purposes since we decided to focus on bivariate models for two outcomes. The remaining 43 reviews were included, and these contained up to 132 studies with two binary outcomes of interest each with a cross classification (two by two) table summarising the number of outcome events and non-events for the treatment and control groups. The relationships between the pair of binary outcomes was either mutually exclusive or an is-subset-of relationship. An is-subset-of relationships refers to when one outcome is contained within the other. For example, the number of patients that have survived with a particular condition at, say, 6 months and a year. A mutually exclusive relationship is when the outcomes are independent of each other and therefore occur separately. An example is death from breast cancer and death from other causes, excluding breast cancer.

For each of the 43 meta-analysis datasets, we used the two by two tables for each outcome in each trial to derive treatment effect estimates (log odds ratio estimates) and corresponding error variances. A fixed 0.5 continuity correction was required if any denominator in the equation for the variance was equal to zero $[16,17]$, that is, if a study had a zero cell in the two by two table then 0.5 was added to all cells for that study. This is a similar approach to the normal approximation analyses in the in the original Trikalinos review $[9,10]$. For a pair of treatment effect estimates in the same trial, we also derived their within-study correlation using the formula provided for an is-subsetof relationship [7, 18], and by Trikalinos \& Olkin for a mutually exclusive relationship [18]. In some studies, the withinstudy correlation was +1 or -1 , which can cause issues of singular variance matrices during the multivariate model estimation. To avoid this issue, we replaced any \pm 1 values with \pm 0.99 ., although other approaches are possible [18].

To each of the 43 meta-analysis datasets, a univariate common-effect meta-analysis was applied to each outcome separately, using maximum likelihood (ML) estimation. Then we also fitted a bivariate common-effect meta-analysis using ML estimation, to jointly analyse both outcomes whilst accounting for any within-study correlations. The ordering of outcome 1 or 2 was irrelevant (i.e. same results obtained regardless), though for the is-subset-of reviews outcome 2 was designated to be the subset of outcome 1.

Following the bivariate analysis, the BoS was quantified for each outcome by calculating the BoS statistic proposed by Jackson et al.:[6]

$$
B o S=100 \% \times\left(1-\frac{\text { variance of summary result from multivariate analysis }}{\text { variance of summary result from univariate analysis }}\right)
$$

The BoS statistic provides the percentage reduction in the variance of a particular summary result that is due to (borrowed from) data from other correlated outcomes. It is the percentage weight toward the summary result for, say, outcome 1 that is given to the study data for other correlated outcomes [6]. For example, in a bivariate meta-analysis, a $B o S$ of $0 \%$ for outcome 1 indicates that the summary result for outcome 1 is based only on data for outcome 1 , whereas a BoS of $100 \%$ indicates that it is based entirely on the correlated data from outcome 2 . The distribution of $B O S$ statistic values was summarised using descriptive statistics and graphically via histograms. 
The process was repeated rather using univariate and bivariate random-effects models, which allow for betweenstudy heterogeneity. Similar conclusions were drawn and so we focus on the results from the common-effect metaanalyses in this paper. Further, some of the bivariate random-effects models suffered from problems estimating the between-study correlation (often 'converged' at -1 or +1 , for reasons explained elsewhere [20]), and so we deemed it more reliable to focus on BoS observed for the bivariate common-effect model.

\section{Examining characteristics associated with BoS}

The following seven meta-analysis level characteristics were selected for examination of their association with BoS statistic values from a bivariate common-effect meta-analysis:

- the percentage of studies with missing data for the outcome of interest

- the percentage of studies with missing data across both outcomes

- the number of studies in the meta-analysis

- the number of studies with only the outcome of interest

- the number of studies with both outcomes

- the average absolute within-study correlation

- the largest absolute within-study correlation

These characteristics were identified by the research team based on analytic reasoning (see Supplementary material), and our previous (applied and methodological) experience $[3,12,15,21]$. The unadjusted effect of each characteristic on the magnitude of BoS was estimated by fitting a linear regression with BoS as the outcome and the characteristic as the only covariate. Two BoS values were available for each of the 43 reviews (one for each outcome), and so the dataset had 86 outcome values in total. A random intercept was used to account for clustering of BoSvalues from the same study. We also considered modelling BoS on the log scale, but this did not change the findings importantly, and therefore we present results on the BoS scale to aid interpretation.

\section{Development and internal validation of a prediction model for BoS}

A multivariable prediction model was developed for predicting BoS in a new bivariate meta-analysis dataset. The 7 characteristics previously listed were candidate predictors for inclusion. As there were 86 BoSvalues for the modelling, this corresponded to 12.3 values per candidate predictor. At the time of model development, this was considered appropriate as it was larger than ten subjects per predictor (often a rule of thumb for sample size), larger than a recent proposal of two values per predictor [22], and ensured a multiplicative margin of error less than $20 \%$ for the residual standard deviation (i.e. lower and upper bounds of $95 \%$ confidence for residual variance within $20 \%$ of the estimated value) $[23,24]$.

A multivariable linear regression model containing all the seven candidate predictors (forcing them all to be included, regardless of statistical significance) was fitted. The apparent model performance was quantified by the apparent $\mathrm{R}^{2}$ statistic. Internal validation was then undertaken to obtain optimism-adjusted estimates of $\mathrm{R}^{2}$ and calibration slope, using bootstrap resampling with 1000 bootstrap samples, as described elsewhere [25-27]. The optimism- 
adjusted calibration slope was then used as a uniform shrinkage factor; that is, we multiplied the predictor effects of the fitted model by the optimism-adjusted calibration slope. Then, forcing the revised predictor effects to be held fixed, we re-estimated the model intercept to ensure calibration-in-the-large. This produced our final model with all predictors.

In addition to fitting full models, a backwards selection procedure was undertaken to identify a simpler model, with pvalues less than 0.1 used to define predictor inclusion. Internal validation and optimism-adjustment was again applied using bootstrapping, which accounted for the variable selection when estimating optimism.

\section{Applications in new data}

For illustration of their potential use, we applied the developed tools to predict BoS in two Cochrane reviews not included in the Trikalinos review, and to three non-Cochrane reviews, with comparison to subsequent multivariate meta-analysis results and observed BoS values.

\section{Results}

\section{BoS values and comparison of bivariate and univariate meta- analysis results}

The 86 BoS statistic values from the 43 meta-analyses in the Trikalinos review are shown alongside the univariate and bivariate meta-analysis results within Figs. 1 and 2 for outcomes 1 and 2, respectively. A large proportion of the BoS values were small; the median (mean) value was $9.0 \%$ (13.2\%), and the inter-quartile range was $2.71-20.18 \%$, with a minimum value of $0.05 \%$ (Figure A in supplementary material). However, 22 of the 86 BoS values were over $20 \%$, and the largest value was $57.2 \%$, indicating how a bivariate meta-analysis provides notably greater efficiency than separate univariate meta-analyses in some applications.

Important differences between bivariate and univariate meta-analysis results arise in some examples, and these tend to occur in situations with largest BoSvalues. For example, for outcome 1 in meta-analysis ID 23 (BoS=35.1\%), the summary log odds ratio was 0.06 (odds ratio $=1.06$ ) from the univariate meta-analysis and -0.15 (odds ratio $=0.86$ ) from the multivariate meta-analysis; hence the direction of the summary effect changed. For outcome 2 in metaanalysis ID 26 ( $B o S=57.2 \%)$, the summary log odds ratio was -1.22 (95\% Cl: -1.33 to -1.11$)$ from the univariate meta-analysis and -0.81 ( $95 \% \mathrm{Cl}$ - -0.88 to -0.73 ) from the multivariate meta-analysis; hence the confidence interval was narrower and the treatment effect less strong after multivariate analysis. Also, for outcome 1 in meta-analysis ID $38(B o S=51.4 \%)$, the summary log odds ratio was $-0.39(95 \% \mathrm{Cl}:-0.81$ to 0.03 ; p-value $=0.068)$ from the univariate meta-analysis and -0.42 ( $95 \% \mathrm{Cl}$ : -0.71 to -0.12 ; $\mathrm{p}$-value $=0.005)$ from the multivariate meta-analysis; hence the statistical significance changed.

\section{Characteristics associated with BoS}

Univariable linear regression analyses found strong evidence that all our seven pre-specified characteristics were positively associated with the magnitude of the BoS statistic value (Table 1). In particular, the amount of missing outcome data and the magnitude of within-study correlation appeared to be important. There was a $0.49 \%$ (95\% Cl: $0.24 \%, 0.74 \%$ ) increase in BoS for every $1 \%$ increase in the percentage of studies missing an outcome, and a $29.08 \%$ (95\% Cl: $16.30 \%, 41.85 \%)$ increase in BoS for an increase in the absolute within-study correlation from 0 to 1 . This is 
sensible, as the amount of BoS has been shown mathematically to depend on the size of correlation and the amount of missing data. $[3,12,15,28]$ Increasing the number of studies was associated with a small increase in BoS.

Table 1

Unadjusted association of seven meta-analysis characteristics and the magnitude of BoS in a bivariate metaanalysis of two binary outcomes

\begin{tabular}{|c|c|c|c|}
\hline \multirow[t]{2}{*}{ Characteristic } & \multicolumn{3}{|c|}{$\begin{array}{l}\text { Unadjusted effect of characteristic on } \\
\text { BoS }\end{array}$} \\
\hline & coefficient & p-value & $95 \% \mathrm{Cl}$ \\
\hline Number of studies & 0.19 & $<0.001$ & 0.09 to 0.29 \\
\hline Number of studies with outcome of interest & 0.17 & 0.013 & 0.04 to 0.31 \\
\hline Number of studies with both outcomes & 0.36 & 0.001 & 0.15 to 0.57 \\
\hline Percentage of studies missing either outcome & 0.49 & $<0.001$ & 0.24 to 0.74 \\
\hline $\begin{array}{l}\text { Percentage of studies missing the outcome corresponding to the BoS } \\
\text { value }\end{array}$ & 0.52 & $<0.001$ & 0.38 to 0.67 \\
\hline Average absolute within-study correlation & 28.36 & $<0.001$ & 14.37 to 42.35 \\
\hline Largest absolute within-study correlation & 29.08 & $<0.001$ & 16.30 to 41.85 \\
\hline
\end{tabular}

\section{Multivariable prediction model for BoS}

Multivariable modelling results are shown in Table 2. After fitting a full model including all seven characteristics, the apparent proportion of variability explained $\left(R^{2}\right)$ was 0.58 . Only four characteristics had strong evidence for an important adjusted association with BoS statistic values: the total number of studies, the number of studies with the outcome of interest, the percentage of studies without the outcome of interest, and the largest absolute within-study correlation value in the meta-analysis dataset. These all had a positive adjusted association with BoSvalues, except for the number of studies providing the outcome of interest which had a negative association. The latter makes sense as, after accounting for the total number of studies, a larger number of studies with the outcome of interest leads to less opportunity for borrowing strength from the correlated outcome. After using backwards selection, only these four characteristics were selected (Table 2), and the model performance was very similar to the full model (apparent $\mathrm{R}^{2}$ = 0.57 ). For parsimony, we therefore chose to use this reduced model going forward. Bootstrapping identified a small amount of overfitting in the final model after variable selection (optimism-adjusted calibration slope $=0.96$; optimism-adjusted $R^{2}=0.50$ ). After applying a global shrinkage factor of 0.96 to adjust for overfitting, the final prediction model was (Table 2):

Predicted BoSfor outcome of interest

$=-13.02+(0.630 *$ number of studies $)$

- $(0.755 *$ number of studies with outcome of interest $)$

$+(0.282 *$ percentage of missing data for outcome of interest $)$

$+(25.581 *$ largest absolute within-study correlation $)$

Equation (1) 
Table 2

Adjusted association of seven meta-analysis characteristics and the magnitude of $B o S$ in a bivariate meta-analysis

\begin{tabular}{|c|c|c|c|}
\hline \multirow[t]{3}{*}{ Characteristic } & $\begin{array}{l}\text { Adjusted effect of } \\
\text { characteristic on } \\
\text { BoS } \\
\text { (full model) }\end{array}$ & $\begin{array}{l}\text { Adjusted effect of } \\
\text { characteristic on } \\
\text { BoS } \\
\text { (after backwards } \\
\text { selection) }\end{array}$ & $\begin{array}{l}\text { Final model after applying } \\
\text { global shrinkage factor of } \\
0.96\end{array}$ \\
\hline & $\begin{array}{l}\text { Coefficient } \\
(95 \% \mathrm{Cl})\end{array}$ & $\begin{array}{l}\text { Coefficient } \\
(95 \% \mathrm{Cl})\end{array}$ & Coefficient \\
\hline & p-value & p-value & \\
\hline \multirow[t]{3}{*}{ Number of studies } & 0.70 & 0.65 & 0.630 \\
\hline & (0.13 to 1.27 ) & (0.19 to 1.12 ) & \\
\hline & 0.017 & 0.006 & \\
\hline \multirow{3}{*}{$\begin{array}{l}\text { Number of studies with outcome of } \\
\text { interest }\end{array}$} & -0.69 & -0.79 & -0.755 \\
\hline & $(-1.39$ to 0.01$)$ & $(-1.38$ to -0.19$)$ & \\
\hline & 0.052 & 0.010 & \\
\hline \multirow{3}{*}{$\begin{array}{l}\text { Number of studies with both } \\
\text { outcomes }\end{array}$} & -0.23 & & \\
\hline & $(-1.20$ to 0.74$)$ & & \\
\hline & 0.64 & & \\
\hline \multirow{3}{*}{$\begin{array}{l}\text { Percentage of studies missing either } \\
\text { outcome }\end{array}$} & -0.12 & & \\
\hline & $(-0.53$ to 0.28$)$ & & \\
\hline & 0.55 & & \\
\hline \multirow{3}{*}{$\begin{array}{l}\text { Percentage of studies missing the } \\
\text { outcome corresponding to the BoS } \\
\text { value }\end{array}$} & 0.35 & 0.29 & 0.282 \\
\hline & (0.09 to 0.61$)$ & (0.10 to 0.48$)$ & \\
\hline & 0.009 & 0.003 & \\
\hline \multirow{3}{*}{$\begin{array}{l}\text { Average absolute within-study } \\
\text { correlation }\end{array}$} & -9.98 & & \\
\hline & $(-33.15$ to 13.19$)$ & & \\
\hline & 0.39 & & \\
\hline \multirow{3}{*}{$\begin{array}{l}\text { Largest absolute within-study } \\
\text { correlation }\end{array}$} & 35.23 & 26.59 & 25.581 \\
\hline & (12.49 to 57.98$)$ & (16.53 to 36.65$)$ & \\
\hline & 0.003 & $<0.001$ & \\
\hline \multirow[t]{3}{*}{ Intercept } & -13.90 & -14.05 & -13.020 \\
\hline & $(-23.22$ to -4.58$)$ & $(-22.85$ to -5.26$)$ & \\
\hline & 0.004 & 0.002 & \\
\hline
\end{tabular}




\begin{tabular}{|llll|}
\hline Characteristic & $\begin{array}{l}\text { Adjusted effect of } \\
\text { characteristic on } \\
\text { BoS } \\
\text { (full model) }\end{array}$ & $\begin{array}{l}\text { Adjusted effect of } \\
\text { characteristic on } \\
\text { BoS } \\
\text { (after backwards } \\
\text { selection) }\end{array}$ & $\begin{array}{l}\text { Final model after applying } \\
\text { global shrinkage factor of } \\
0.96\end{array}$ \\
\cline { 2 - 3 } & $\begin{array}{l}\text { Coefficient } \\
\text { Coefficient } \\
(95 \% \mathrm{Cl})\end{array}$ & $\begin{array}{l}(95 \% \mathrm{Cl}) \\
\text { Coefficient }\end{array}$ \\
\hline M-value & $\mathrm{p}$-value & \\
\hline Apparent & Apparent & $\begin{array}{l}\text { Optimism-adjusted } \mathrm{R}^{2}= \\
0.50\end{array}$ \\
\hline $\mathrm{R}^{2}=0.58$ & $\mathrm{R}^{2}=0.57$ & \\
\hline
\end{tabular}

A scatter plot of the observed BoS values against the predicted BoS values (before and after shrinkage) is shown in Fig. 3. The larger the predicted $B o S$ then the greater the rationale for undertaking multivariate meta-analysis. We suggest a threshold of about 15 to $20 \%$ for flagging that a multivariate meta-analysis is worth considering. Note that the equation depends on knowing the largest within-study correlation. If this is not known, we suggest assuming a large value, say 0.8 , for the largest absolute within-study correlation. This would then reveal the predicted BoS in a situation where the two outcomes are highly correlated.

\section{Applied examples}

We applied Eq. (1) to predict BoS in two Cochrane reviews published in 2017 by Buzzetti et al.[29] and Feinberg et al. [30], which both contained an is-a-subset-of relationship between two outcomes. Buzzetti et al. reviewed randomised trials comparing the use of glucocorticosteriods for alcoholic hepatitis compared with no intervention, and the two correlated outcomes were mortality at maximal follow-up and mortality at 30 days.[29] Feinberg et al. reviewed randomised trials that compared experimental nutrition support to a control for disease-related malnutrition in Intensive Care Unit participants, including trauma, characterised as "at nutritional risk"; their two correlated outcomes of interest were all cause mortality at the end of intervention and at maximum follow-up.[30]

The results are shown in Table 3. For the Buzzetti et al. review, using Eq. (1) the predicted BoS is only $10.8 \%$ for outcome 1 , but larger at $20.1 \%$ for outcome 2 as calculated below.

Predicted BoSfor outcome of interest

$=-13.02+(0.630 *$ number of studies $)$

- $(0.755 *$ number of studies with outcome of interest $)$

$+(0.282 *$ percentage of missing data for outcome of interest $)$

$+(25.581 *$ largest absolute within-study correlation)

$=-13.02+(0.630 * 12)$

$-(0.755 * 9)$

$+(0.282 * 25)$ 
$+(25.581 * 0.99)$

\section{$=20.1 \%$}

Given the predicted BoSfor outcome 2 is moderately large, it flags that a multivariate meta-analysis may be useful in this review, for outcome 2 to borrow strength from outcome 1. Subsequently, we applied a multivariate meta-analysis and the summary odds ratio for outcome 2 was 0.89 (95\% Cl: 0.69 to 1.15), which provided much weaker evidence of a beneficial treatment effect than the univariate meta-analysis results (summary odds ratio $=0.69,95 \% \mathrm{Cl}$ : 0.48 to 0.98). Indeed, the actual observed BoS was $47.7 \%$ for outcome 2, and so our predicted BoS of $20.1 \%$ was an underestimate. Nevertheless, the predicted BoS still flagged that a multivariate meta-analysis was potentially important. For outcome 1, the observed BoS was 3.2\%, and so multivariate and univariate meta-analysis results were similar, as anticipated by the lower predicted BoS value of $10.8 \%$.

In the Feinberg et al. review, for outcome 1 the predicted $B o S$ was $21 \%$, again flagging that a multivariate approach may be worth the extra effort for outcome 1 in this review. When applying the multivariate model, the observed BoS was $32.8 \%$ for outcome 1 , and this led to a narrower confidence interval for the summary treatment effect compared to univariate meta-analysis for outcome 1 (Table 3), although no change in statistical or clinical significance (unlike the Buzzetti et al. review). 
Table 3

True and predicted BoS values for two Cochrane reviews, alongside their multivariate and univariate meta-analysis results

\begin{tabular}{|c|c|c|c|c|c|c|c|c|}
\hline \multirow{2}{*}{$\begin{array}{l}\text { Review } \\
\text { (total } \\
\text { number } \\
\text { of } \\
\text { studies) }\end{array}$} & \multirow[t]{2}{*}{ Outcome } & \multirow[t]{2}{*}{$\begin{array}{l}\text { Number } \\
\text { of } \\
\text { studies } \\
\text { with the } \\
\text { outcome }\end{array}$} & \multirow[t]{2}{*}{$\begin{array}{l}\text { Predicted } \\
\text { BoS from } \\
\text { Eq. (1) }\end{array}$} & \multirow[t]{2}{*}{$\begin{array}{l}\text { Observed } \\
\text { BoS after } \\
\text { bivariate } \\
\text { meta- } \\
\text { analysis }\end{array}$} & \multicolumn{2}{|c|}{$\begin{array}{l}\text { Bivariate common- } \\
\text { effect meta-analysis }\end{array}$} & \multicolumn{2}{|c|}{$\begin{array}{l}\text { Univariate common- } \\
\text { effect meta-analysis } \\
\text { for each outcome } \\
\text { separately }\end{array}$} \\
\hline & & & & & $\begin{array}{l}\text { Summary } \\
\text { odds } \\
\text { ratio } \\
(95 \% \mathrm{Cl})\end{array}$ & $\begin{array}{l}\text { Standard } \\
\text { error of } \\
\text { log odds } \\
\text { ratio }\end{array}$ & $\begin{array}{l}\text { Summary } \\
\text { odds } \\
\text { ratio } \\
(95 \% \mathrm{Cl})\end{array}$ & $\begin{array}{l}\text { Standard } \\
\text { error of } \\
\text { log odds } \\
\text { ratio }\end{array}$ \\
\hline \multirow{4}{*}{$\begin{array}{l}\text { Buzzetti } \\
\text { et al.[29] } \\
(12)\end{array}$} & 1: & 9 & $20.1 \%$ & $47.7 \%$ & 0.89 & 0.13 & 0.69 & 0.18 \\
\hline & $\begin{array}{l}\text { Mortality at } \\
30 \text { days }\end{array}$ & & & & $\begin{array}{l}(0.69 \text { to } \\
1.15)\end{array}$ & & $\begin{array}{l}(0.48 \text { to } \\
0.98)\end{array}$ & \\
\hline & $2:$ & 12 & $10.8 \%$ & $3.2 \%$ & 0.89 & 0.13 & 0.91 & 0.13 \\
\hline & $\begin{array}{l}\text { Mortality at } \\
\text { maximal } \\
\text { follow-up }\end{array}$ & & & & $\begin{array}{l}(0.69 \text { to } \\
1.14)\end{array}$ & & $\begin{array}{l}(0.70 \text { to } \\
1.17)\end{array}$ & \\
\hline \multirow{4}{*}{$\begin{array}{l}\text { Feinberg } \\
\text { et al.[30] } \\
\text { 15) }\end{array}$} & 1: & 11 & $21.0 \%$ & $32.8 \%$ & 0.97 & 0.09 & 0.97 & 0.11 \\
\hline & $\begin{array}{l}\text { All-cause } \\
\text { mortality at } \\
\text { end of } \\
\text { intervention }\end{array}$ & & & & $\begin{array}{l}(0.81 \text { to } \\
1.16)\end{array}$ & & $\begin{array}{l}(0.78 \text { to } \\
1.20)\end{array}$ & \\
\hline & $2:$ & 15 & $10.4 \%$ & $0.2 \%$ & 0.96 & 0.08 & 0.96 & 0.08 \\
\hline & $\begin{array}{l}\text { All-cause } \\
\text { mortality at } \\
\text { maximum } \\
\text { follow-up }\end{array}$ & & & & $\begin{array}{l}(0.82 \text { to } \\
1.12)\end{array}$ & & $\begin{array}{l}(0.82 \text { to } \\
1.13)\end{array}$ & \\
\hline
\end{tabular}

\section{Discussion}

Key findings and recommendations

Methods for multivariate meta-analysis of multiple correlated outcomes are more complex for non-statisticians and may not always be worth the extra effort for Cochrane and other reviewers. However, on occasion the multivariate approach provides important extra efficiency compared to a conventional univariate meta-analysis of each outcome separately and may even lead to different statistical or clinical conclusions. Our empirical examination using the datasets from the Trikalinos review shows that such situations occur mainly when the borrowing of strength amongst outcomes is large. Hence, flagging to (non-statistical) researchers in advance of data analysis the predicted magnitude of BoS is potentially helpful.

We identified four characteristics as strongly associated with BoS in Cochrane intervention reviews with multiple binary outcomes: the total number of studies, the number of studies with the outcome of interest, the percentage of studies missing the outcome of interest, and the largest absolute within-study correlation. Based on these we developed prediction Eq. (1), which can help predict BoS for outcomes in a new Cochrane review. In particular, if this equation predicts $B o S$ to be moderate or large (say greater than about 15 to $20 \%$ ) then it may motivate Cochrane 
reviewers to obtain additional statistical support to undertake the multivariate approach, and to invest time and resources trying to extract or derive within-study correlations amongst outcomes, or even to obtain individual participant data from studies to estimate them directly [31]. It should also be noted that, even if BoS is anticipated to be low, sometimes there are other reasons why a multivariate meta-analysis is needed; for example, if functions of summary results are required $[9,10,12,15]$, or if the actual estimate(s) of correlation are of interest.

\section{Limitations}

There are limitations of our work. Although the developed prediction equation explained $50 \%$ of the variation in $B o S$ values, there can still be a reasonable discrepancy between predicted and observed Bos values (see Fig. 3 and Table 3). However, the model is not intended to perfectly predict BoS, and is rather intended as a tool to provide additional insight for when the multivariate approach is worth considering. This was illustrated in our two applied examples, where outcomes with predicted BoSvalues of $>20 \%$ suggested multivariate meta-analysis may be useful, and subsequently applying multivariate meta-analysis improved precision and, in one example, even changed the statistical and clinical conclusions.

Another limitation is that the identified predictors of BoS are based on Cochrane reviews of binary outcomes, and so further evaluation and extension is needed for other settings. Also, our prediction model equation requires the researcher to input the largest absolute within-study correlation; this might not be available and so, as mentioned previously, we suggest assuming a large value such as 0.8 , to predict $B o S$ assuming correlations are high.

The work presented is based on assuming common treatment effects. BoS results were also obtained in our examples after using random-effects models to allow for heterogeneity, and similar conclusions found. However, predicting $B o S$ in a random effects setting is more complex, due to the impact of between-study variances and between-study correlations, which are difficult to gauge in advance, although informative prior distributions could be considered [32, 33]. Furthermore, BoS is harder to define in random effects situations, as the univariate meta-analysis must be forced to have the same between-study variance estimates as the bivariate meta-analysis, to make comparisons fair $[6,21]$. Hence, we consider it simpler for researchers to focus on predicting BoS initially assuming no heterogeneity.

Summary

Though multivariate meta-analysis is a more complex evidence synthesis method, it may sometimes be important to consider in Cochrane reviews of multiple outcomes. We have identified key characteristics and developed a prediction model to help to flag when a multivariate meta-analysis may be beneficial to Cochrane reviewers with multiple binary outcomes. Extension to non-Cochrane reviews and other outcome types is still required.

\section{What This Study Adds}

- Multivariate meta-analysis jointly synthesises multiple correlated effect estimates from multiple studies, which enables borrowing of strength across outcomes, which can sometimes lead to difference to results from a standard univariate meta-analysis.

- Our empirical examination of Cochrane intervention reviews with multiple binary outcomes shows that multivariate meta-analysis is most influential when the borrowing of strength (BoS) amongst outcomes is large.

- Four characteristics were strongly associated with BoS. the total number of studies, the number of studies with the outcome of interest, the percentage of studies missing the outcome of interest, and the largest absolute 
within-study correlation.

- We developed a prediction equation based on these characteristics, to help predict BoSfor outcomes in a new Cochrane review, so to flag when the multivariate approach may be worth considering instead of separate univariate analyses.

- We suggest that if this equation predicts BoS to be moderate or large (say greater than about 15 to $20 \%$ ) then it may motivate Cochrane reviewers to undertake the multivariate meta-analysis approach.

\section{Declarations}

Ethics approval and consent to participate:

Not applicable.

\section{Consent for publication:}

Not applicable.

\section{Availability of data and materials:}

The datasets used and/or analysed during the current study are available from the corresponding author on reasonable request.

\section{Competing interests and Funding Statement:}

Miriam Hattle was funded by a Keele University Acorn PhD studentship. This paper presents independent research funded by the National Institute for Health Research (NIHR) under its Programme Grants for Applied Research Programme (Reference Number RP-PG-0615-20002), with co-funding from Arthritis Research UK. Richard Riley is supported by funding from the Evidence Synthesis Working Group, which is funded by the NIHR School for Primary Care Research (NIHR SPCR) [ProjectNumber 390]. The views expressed are those of the authors and not necessarily those of the NHS, the NIHR or the Department of Health and Social Care. Yong Chen was supported in part by grant number 1R01LM012607 from the National Library of Medicine and 1R01Al130460 from the National Institute of Allergy and Infectious Diseases.

\section{Author's contributions:}

$\mathrm{RR}, \mathrm{DB}$ and $\mathrm{MH}$ planned the research question and study design. $\mathrm{MH}$ performed all the analyses using data originally extracted by TT and CS. All authors provided feedback on the methods and results. MH and RR drafted the paper, and then revised following feedback and comments from all authors.

\section{Acknowledgements:}

Not applicable

\section{References}

1. Riley RD, Gates SG, Neilson J, Alfirevic Z. Statistical methods can be improved within Cochrane Pregnancy and Childbirth Reviews. J Clin Epidemiol. 2011;64(6):608-18. 
2. Van Houwelingen HC, Arends LR, Stijnen T. Advanced methods in meta-analysis: multivariate approach and meta-regression. Stat Med. 2002;21:589-624.

3. Jackson D, Riley RD, White IR. Multivariate meta-analysis: potential and promise. Stat Med. 2011;30:2481-98.

4. Kirkham JJ, Riley RD, Williamson PR. A multivariate meta-analysis approach for reducing the impact of outcome reporting bias in systematic reviews. Stat Med. 2012;31:2179-95.

5. Higgins JP, Whitehead A. Borrowing strength from external trials in a meta-analysis. Stat Med. 1996;15:2733-49.

6. Jackson D, White IR, Price M, Copas J, Riley RD. Borrowing of strength and study weights in multivariate and network meta-analysis. Stat Methods Med Res. 2017;26:2853-68.

7. Wei Y, Higgins JP. Estimating within-study covariances in multivariate meta-analysis with multiple outcomes. Stat Med. 2013;32:1191-205.

8. Riley RD, Thompson JR, Abrams KR. An alternative model for bivariate random-effects meta-analysis when the within-study correlations are unknown. Biostatistics. 2008;9:172-86.

9. Trikalinos TA, Hoaglin DC, Schmid CH. An empirical comparison of univariate and multivariate meta-analyses for categorical outcomes. Stat Med. 2014;33:1441-59.

10. Trikalinos TA, Hoaglin DC, Schmid CH. Empirical and Simulation-Based Comparison of Univariate and Multivariate Meta-Analysis for Binary Outcomes. Methods Research Report. Agency for Healthcare Research and Quality. 2013:Publication No. 13-EHC066-EF.

11. Jones AP, Riley RD, Williamson PR, Whitehead A. Meta-analysis of individual patient data versus aggregate data from longitudinal clinical trials. Clin Trials. 2009;6:16-27.

12. Riley RD. Multivariate meta-analysis: the effect of ignoring within-study correlation. J R Stat Soc A Stat Soc. $2009 ; 172$.

13. Price MJ, Blake HA, Kenyon S, White IR, Jackson D, Kirkham JJ, et al. Empirical comparison of univariate and multivariate meta-analyses in Cochrane Pregnancy and Childbirth reviews with multiple binary outcomes. Res Synth Methods. 2019;10:440-51.

14. Moran JL. Multivariate meta-analysis of critical care meta-analyses: a meta-epidemiological study. BMC Med Res Methodol. 2021;21:148.

15. Riley RD, Abrams KR, Lambert PC, Sutton AJ, Thompson JR. An evaluation of bivariate random-effects metaanalysis for the joint synthesis of two correlated outcomes. Stat Med. 2007;26:78-97.

16. Sweeting MJ, Sutton AJ, Lambert PC. What to add to nothing? Use and avoidance of continuity corrections in meta-analysis of sparse data. Stat Med. 2004;23:1351-75.

17. Higgins JPT, Green SB. Cochrane Handbook for Systematic Reviews of Interventions Version 5.1.0 [updated March 2011]: The Cochrane Collaboration (Available from www.handbook.cochrane.org); 2011.

18. Trikalinos TA, Olkin I. Meta-analysis of effect sizes reported at multiple time points: A multivariate approach. Clin Trials. 2012.

19. Trikalinos TA, Olkin I. A method for the meta-analysis of mutually exclusive binary outcomes. Stat Med. 2008;27:4279-300.

20. Riley RD, Abrams KR, Sutton AJ, Lambert PC, Thompson JR. Bivariate random-effects meta-analysis and the estimation of between-study correlation. BMC Med Res Methodol. 2007;7:3.

21. Copas JB, Jackson D, White IR, Riley RD. The role of secondary outcomes in multivariate meta-analysis. J Roy Stat Soc: Ser C (Appl Stat). 2018;67:1177-205. 
22. Austin PC, Steyerberg EW. The number of subjects per variable required in linear regression analyses. J Clin Epidemiol. 2015;68:627-36.

23. Harrell FE, Jr. Regression Modeling Strategies: With Applications to Linear Models, Logistic and Ordinal Regression, and Survival Analysis (Second Edition). New York: Springer; 2015.

24. Riley RD, Ensor J, Snell KIE, Harrell FE Jr, Martin GP, Reitsma JB, et al. Calculating the sample size required for developing a clinical prediction model. BMJ. 2020;368:m441.

25. Steyerberg EW. Clinical prediction models: a practical approach to development, validation, and updating. New York: Springer; 2009.

26. Moons KG, Altman DG, Reitsma JB, loannidis JP, Macaskill P, Steyerberg EW, et al. Transparent Reporting of a multivariable prediction model for Individual Prognosis Or Diagnosis (TRIPOD): explanation and elaboration. Ann Intern Med. 2015;162:W1-73.

27. Riley RD, van der Windt D, Croft P, Moons KGM. Prognosis Research in Healthcare: Concepts, Methods and Impact. Oxford: Oxford University Press; 2019.

28. Riley RD, Jackson D, Salanti G, Burke DL, Price M, Kirkham J, et al. Multivariate and network meta-analysis of multiple outcomes and multiple treatments: rationale, concepts, and examples. BMJ. 2017;358:j3932.

29. Buzzetti E, Kalafateli M, Thorburn D, Davidson BR, Thiele M, Gluud LL, et al. Pharmacological interventions for alcoholic liver disease (alcohol-related liver disease): an attempted network meta-analysis. Cochrane Database Syst Rev. 2017;3:CD011646.

30. Feinberg J, Nielsen EE, Korang SK, Halberg Engell K, Nielsen MS, Zhang K, et al. Nutrition support in hospitalised adults at nutritional risk. Cochrane Database Syst Rev. 2017;5:CD011598.

31. Riley RD, Price MJ, Jackson D, Wardle M, Gueyffier F, Wang J, et al. Multivariate meta-analysis using individual participant data. Res Synth Method. 2015;6:157-74.

32. Burke DL, Bujkiewicz S, Riley RD. Bayesian bivariate meta-analysis of correlated effects: Impact of the prior distributions on the between-study correlation, borrowing of strength, and joint inferences. Stat Methods Med Res. 2018;27:428-50.

33. Turner RM, Davey J, Clarke MJ, Thompson SG, Higgins JP. Predicting the extent of heterogeneity in metaanalysis, using empirical data from the Cochrane Database of Systematic Reviews. Int J Epidemiol. 2012;41:818-27.

\section{Figures}




\section{Outcome 1 \\ ML fixed-effect meta-analysis}

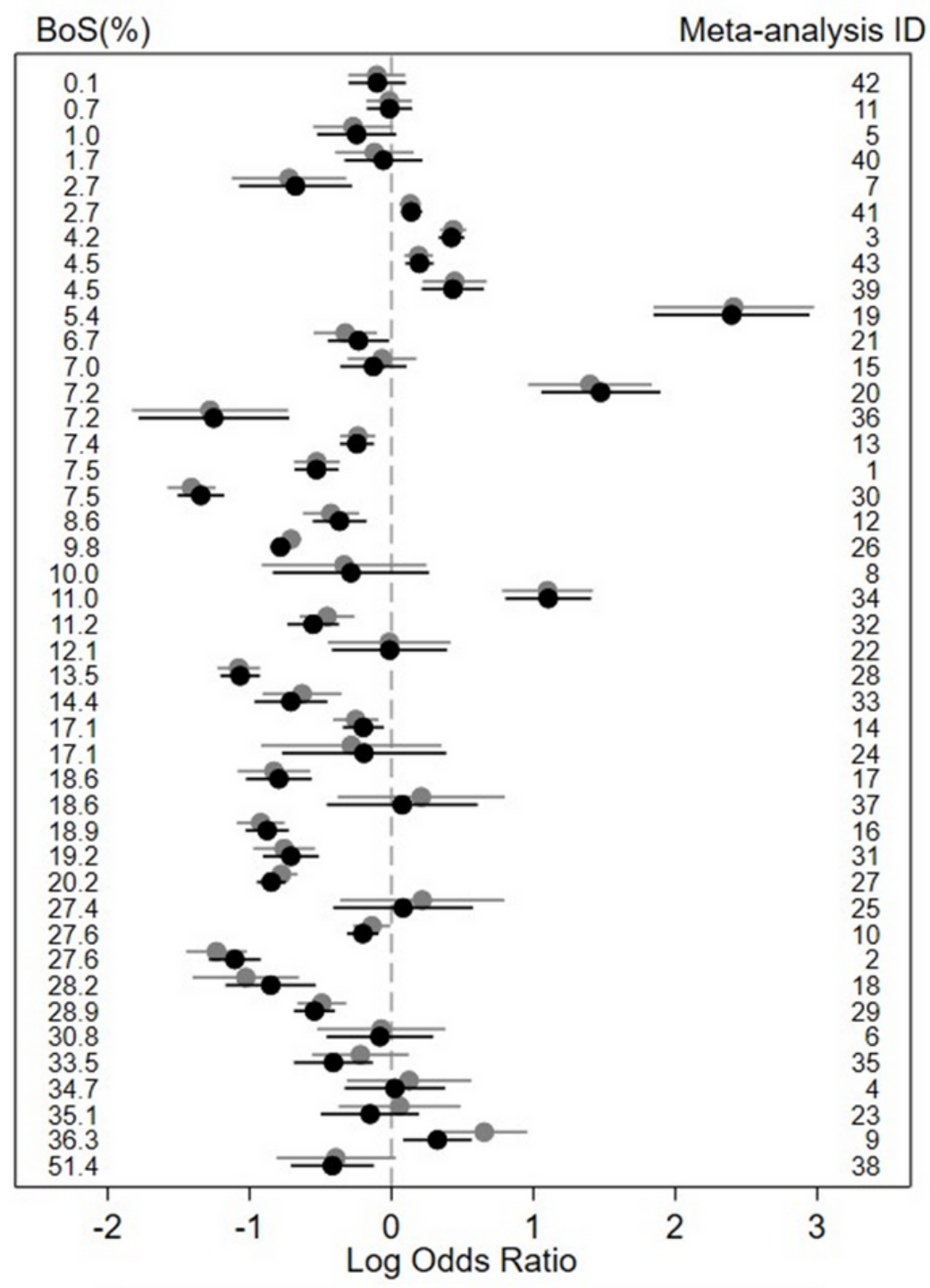

$$
\begin{array}{ll}
\text { - Univariate } & \mathrm{Cl} \text { for Univariate } \\
\text { - Multivariate } & \mathrm{Cl} \text { for Multivariate }
\end{array}
$$

Figure 1

Comparison of the univariate and bivariate meta-analysis results on the log odds ratio scale for outcome 1 from the 43 reviews examined by Trikalinos et al, ordered by the magnitude of the BoS statistic 


\section{Outcome 2 \\ ML fixed-effect meta-analysis}

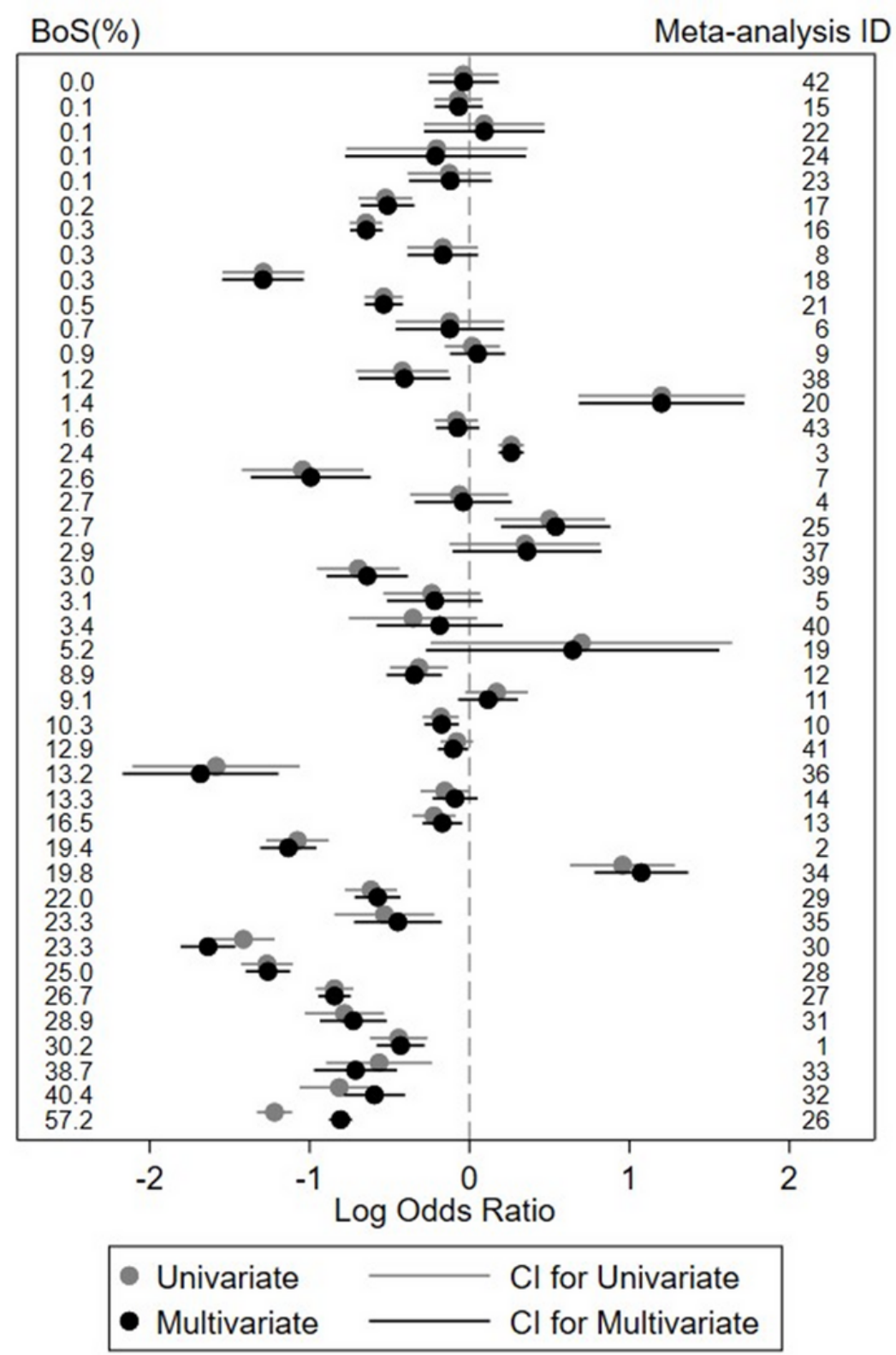

Figure 2

Comparison of the univariate and bivariate meta-analysis results on the log odds ratio scale for outcome 2 for the 43 meta-analysis examined by Trikalinos et al, ordered by the magnitude of the BoS statistic 


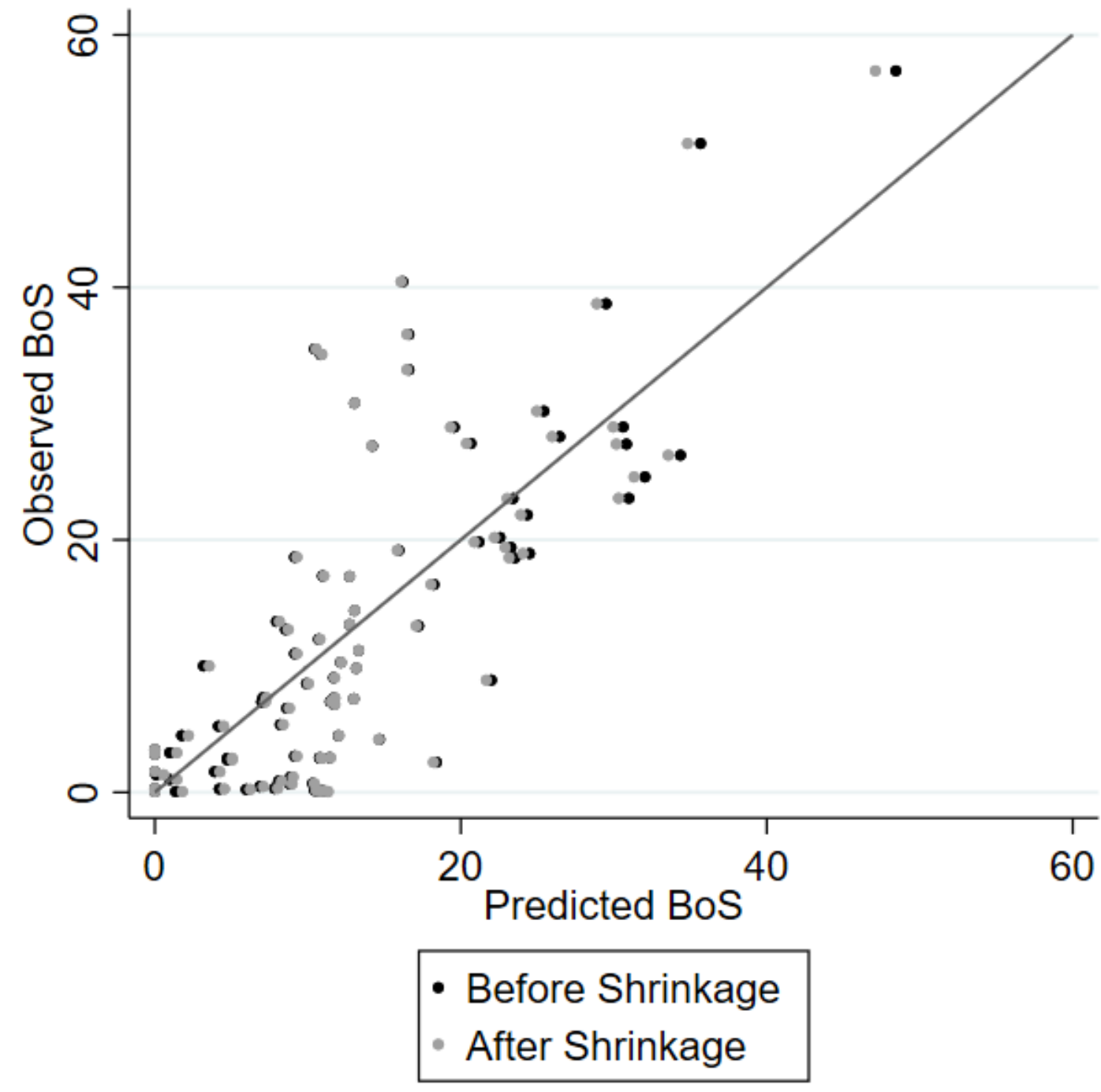

Figure 3

Scatter plot of the observed BoS versus the predicted BoS from the multivariable model after backwards selection (before and after shrinkage)

\section{Supplementary Files}

This is a list of supplementary files associated with this preprint. Click to download.

- Supplementarymaterial.docx 